\title{
Microscopy and spectroscopy of interactions between metallopolymers and carbon nanotubes
}

Fiona Frehill ${ }^{1}$, Marc in het Panhuis ${ }^{2, *}$, Nigel A. Young ${ }^{2}$, William Henry ${ }^{1}$, Johan Hjelm ${ }^{1}$ and Johannes

G. $\operatorname{Vos}^{1}$

1. National Centre for Sensor Research, School of Chemical Sciences, Dublin City University, Dublin 9, Ireland.

2. Department of Chemistry, the University of Hull, Hull, HU6 7RX, United Kingdom.

* Author to whom correspondence should be addressed: M.Panhuis@hull.ac.uk 


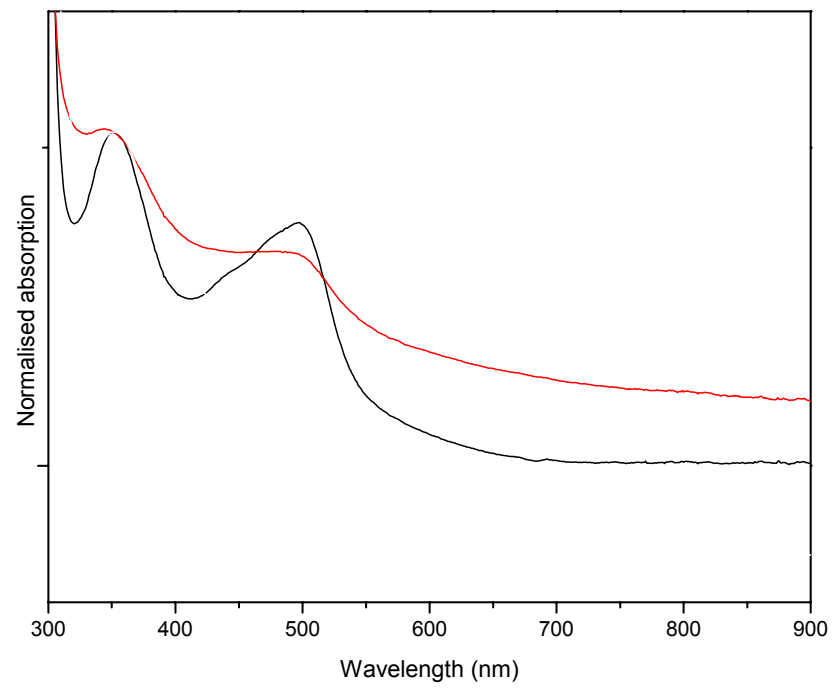

Figure S1. UV-visible absorption spectra of Ru-metallopolymer, $\left[\mathrm{Ru}(\mathrm{bpy})_{2} \mathrm{PVP}{ }_{10} \mathrm{Cl}\right] \mathrm{Cl}$ (black line), and composite 1 (red line, MWNT) normalised to highest energy peak.

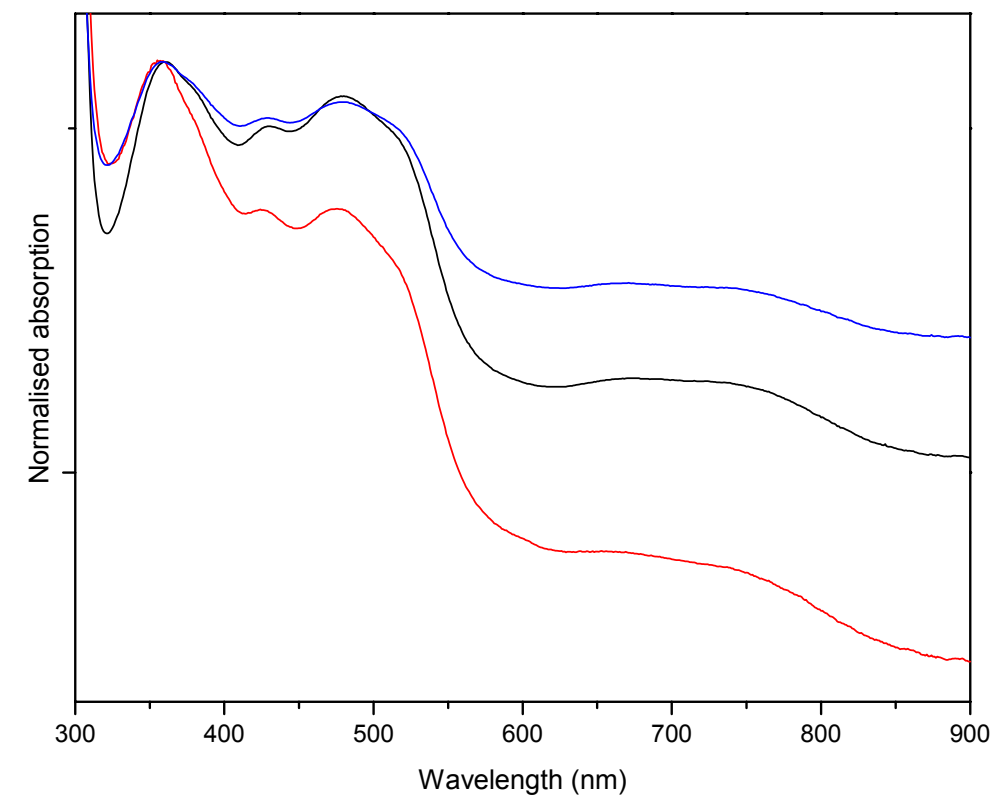

Figure S2. UV-visible absorption spectra of Os-metallopolymer $\left[\mathrm{Os}(\mathrm{bpy}){ }_{2} \mathrm{PVP}_{10} \mathrm{Cl}\right] \mathrm{Cl}$ (black line), composite $\mathbf{3}$ (red line, MWNT) and 4 (blue line) normalised to highest energy peak. 

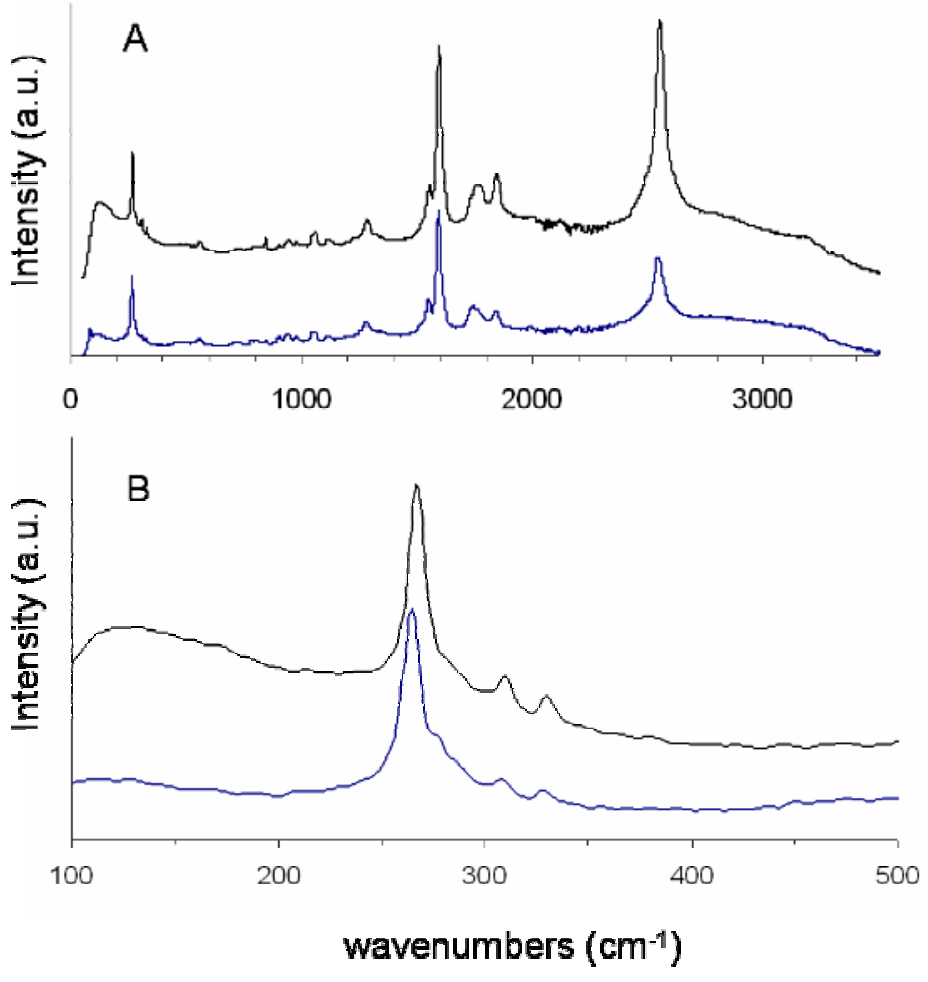

Figure S3. Raman spectra (1064 nm excitation) of HiPco SWNT (blue line) and composite 2 (black line) samples. (A) Full spectra showing radial breathing modes (RBM), D-band, G-bands and twophonon-scattering features and (B) radial breathing modes features. 


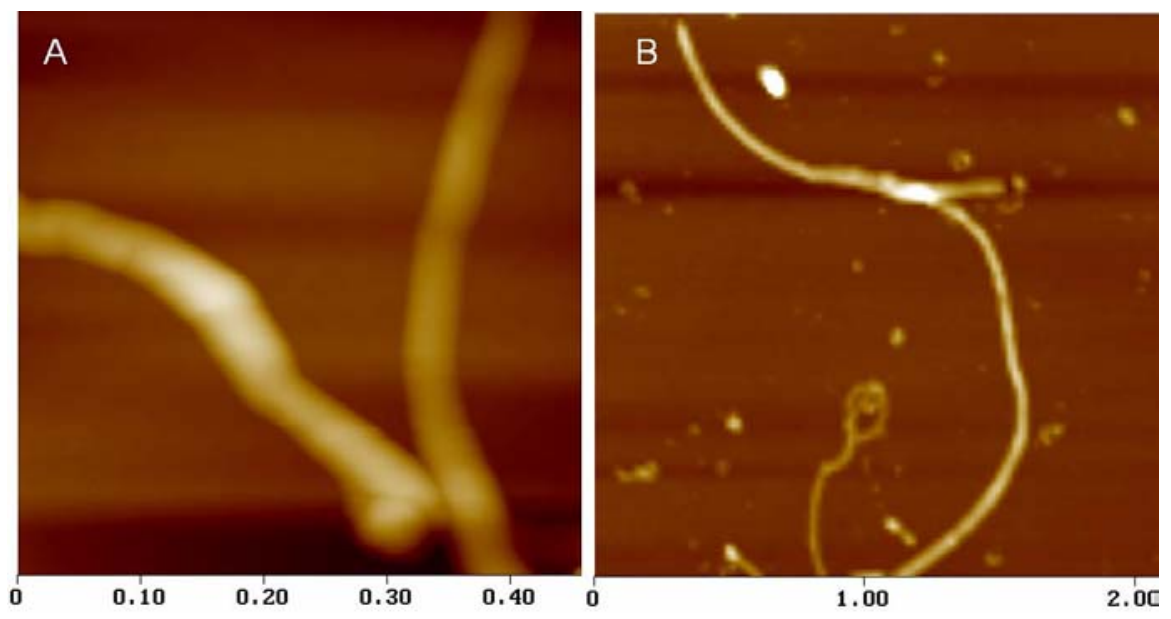

Figure S4. Tapping mode atomic force microscopy (AFM) images of MWNT on amino-silanised $\mathrm{SiO}_{2}$ wafer after sanilinisation treatment and washing with Milli-Q water, with image dimensions: (A) 0.4584 $\mu \mathrm{m} \times 0.4584 \mu \mathrm{m}, \mathrm{z}$-range $=75 \mathrm{~nm}$ and (B) $2.090 \mu \mathrm{m} \times 2.090 \mu \mathrm{m}, \mathrm{z}$-range $=50 \mathrm{~nm}$.

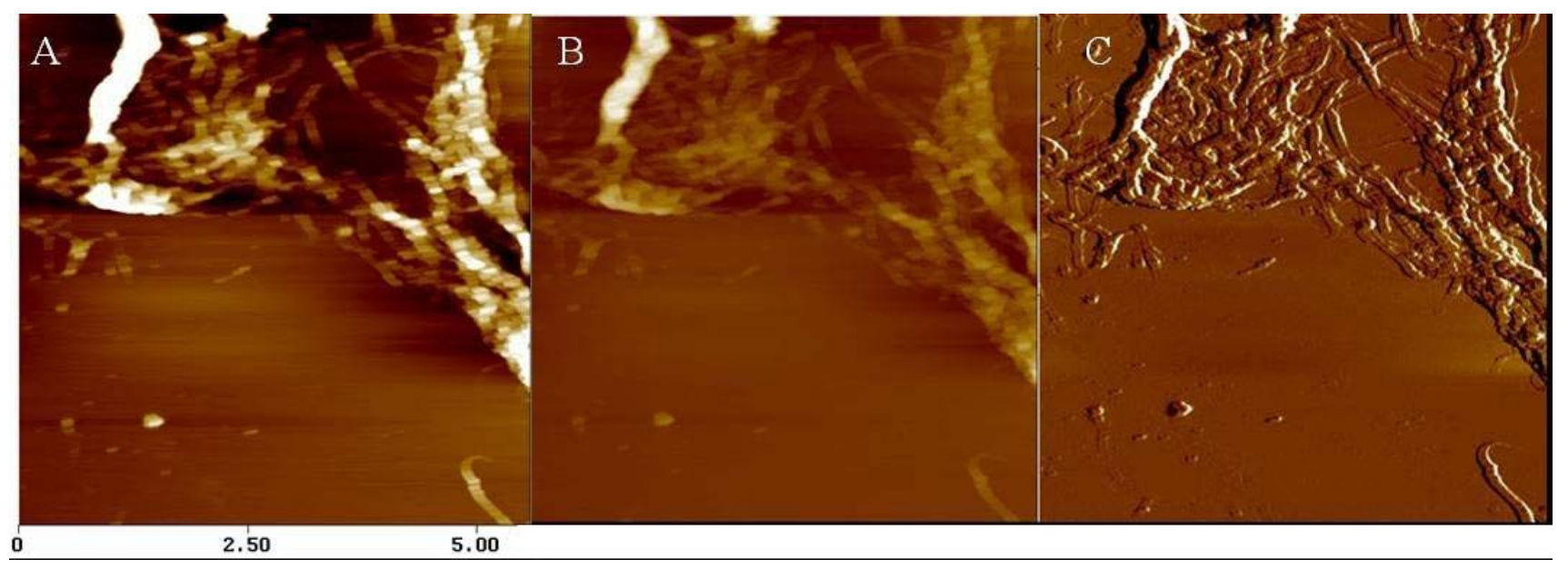

Figure S5. Tapping mode AFM images for composite 2 (Ru-metallopolymer + SWNT) after sanilinisation treatment and washing with Milli-Q water. Height data $5.586 \mu \mathrm{m}$ x $5.586 \mu \mathrm{m}$ image with $\mathrm{z}$ range $=(\mathrm{A}) 100 \mathrm{~nm}$; (B) $300 \mathrm{~nm}$. (C) Amplitude data. 


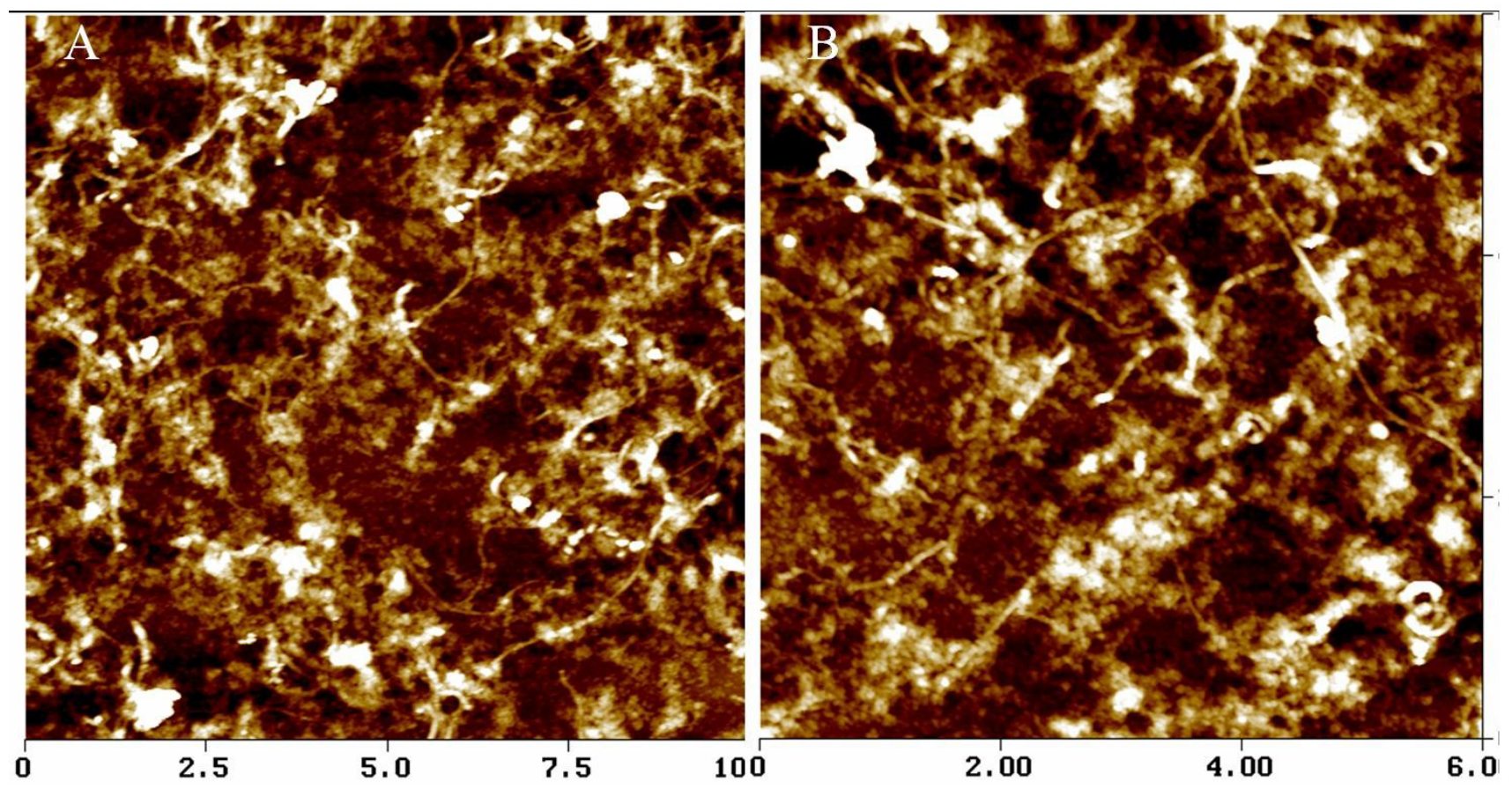

Figure S6. Tapping mode AFM images for composite 3 (Os-metallopolymer + MWNT). Composite solution was allowed to evaporate slowly over a $24 \mathrm{hr}$ period. (A) $10.0 \mu \mathrm{m}$ x $10.0 \mu \mathrm{m}$ image; (B) $6 \mu \mathrm{m} \times$ $6.0 \mu \mathrm{m}, \mathrm{z}$ range $=75 \mathrm{~nm}$.

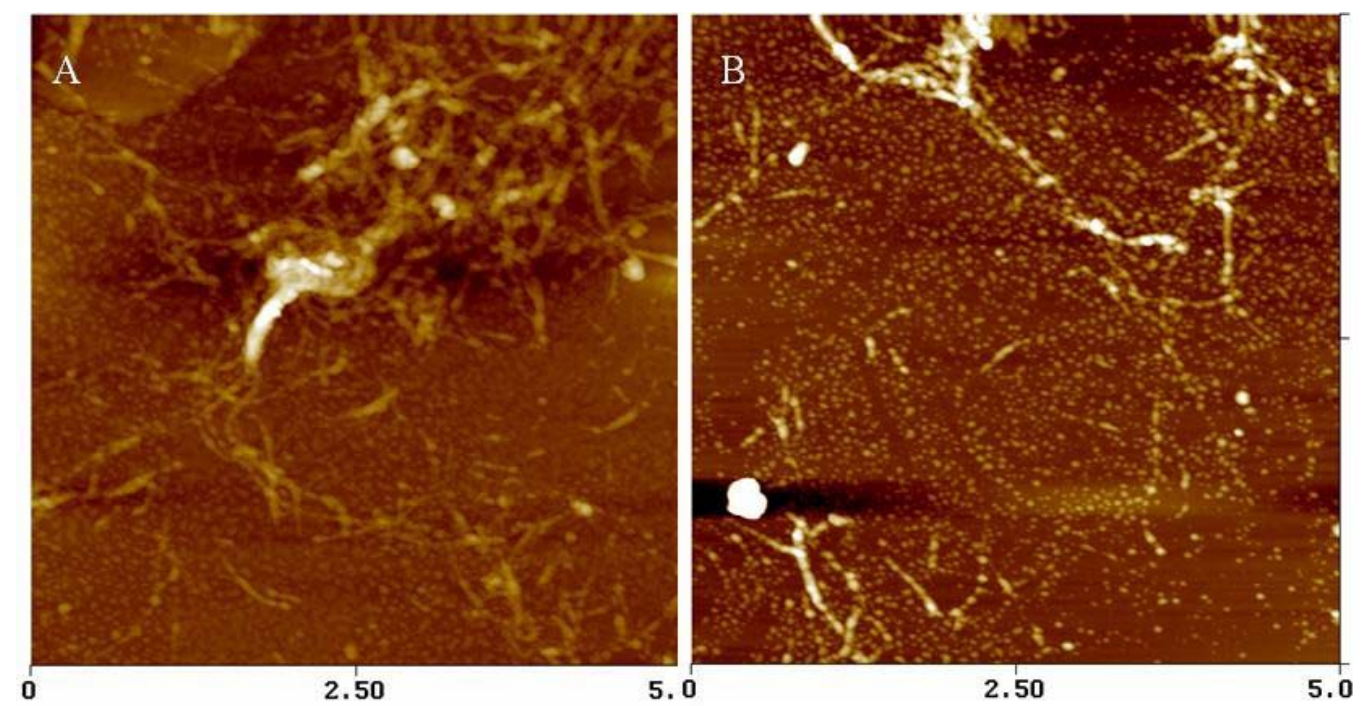

Figure S7. Tapping mode AFM images for composite 4 (Os-metallopolymer + SWNT) after sanilinisation treatment and washing with Milli-Q water.. $5.0 \mu \mathrm{m} \times 5.0 \mu \mathrm{m}$ (A) $\mathrm{z}=125 \mathrm{~nm}$; (B) $\mathrm{z}=75$ $\mathrm{nm}$. 